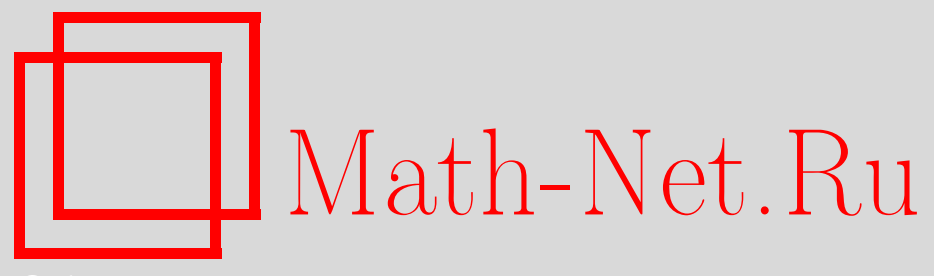

С. Ю. Доброхотов, М. Руло, Квазиклассический аналог принципа Мопертюи-Якоби и его приложение к линейной теории волн на воде, Матем. заметки, 2010, том 87, выпуск 3, 458-463

DOI: https://doi.org/10.4213/mzm8675

Использование Общероссийского математического портала Math-Net.Ru подразумевает, что вы прочитали и согласны с пользовательским соглашением http: //www . mathnet.ru/rus/agreement

Параметры загрузки:

IP: 54.162 .127 .20

26 апреля 2023 г., 14:35:05

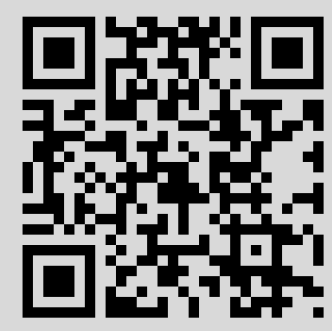




\section{Квазиклассический аналог принципа Мопертюи-Якоби и его приложение к линейной теории волн на воде}

\section{С. Ю. Доброхотов, М. Руло}

1. Введение. Пусть $M-d$-мерное гладкое многообразие, $T^{*} M-$ фазовое пространство с (локальными) координатами

$$
y=(p, x), \quad p={ }^{t}\left(p_{1}, \ldots, p_{d}\right), \quad x={ }^{t}\left(x_{1}, \ldots, x_{d}\right) .
$$

Предположим, что на $T^{*} M$ задано семейство гладко зависящих от параметров $J=$ $\left(J_{1}, \ldots, J_{d}\right)$ лагранжевых многообразий $\Lambda(J)=\{p=P(\varphi, J), x=X(\varphi, J)\}$, диффеоморфных тору

$$
\mathbb{T}^{d}=\mathbb{R}^{d} / 2 \pi \mathbb{Z}^{d}, \quad J=\left(J_{1}, \ldots, J_{d}\right) \in \mathscr{M} .
$$

Здесь $\varphi=\left(\varphi_{1}, \ldots, \varphi_{d}\right), \varphi_{j} \in \mathbf{S}^{1},-$ угловые координаты на $\Lambda(J)$, задающие полуплотность $\left|d \varphi_{1} \wedge \cdots \wedge d \varphi_{d}\right|^{1 / 2}, \mathscr{M}$ - некоторая область в $\mathbb{R}^{d}$. Пусть $\gamma_{j}, j=1, \ldots, d,-$ базисные циклы на $\Lambda(J)$. Они определяют переменные действия, задаваемые в канонических координатах $(x, p)$ формулами $I_{j}=\oint_{\gamma_{j}} p d x$, и, кроме того, задают индексы Маслова $m_{j}$. В дальнейшем всегда будем считать, что в качестве параметров $J_{j}$ выбраны действия $I_{j}$ и что переменные действия $J$ и углы $\varphi$ на $\Lambda(J)$ образуют симплектические сопряженные координаты (вообще говоря, локальные). В силу предположения о гладкой зависимости $\Lambda(J)$ от параметров можно считать, что циклы $\gamma_{j}$ гладко зависят от $J \in \mathscr{M}$, поэтому и индексы Маслова $m_{j}$ оказываются одинаковыми для $\gamma_{j}$ с фиксированным $j$. Зафиксируем на $\Lambda(J)$ некоторые (отмеченные) точки $r(J)$. Предположим далее, что на $\Lambda(J)$ заданы гладкие функции $\chi(\varphi, J)$, также гладко зависящие от $J$. Пусть $h$ - малый положительный параметр. Для каждого фиксированного $h$ построим множество $\mathscr{M}_{q} \in \mathscr{M}$, состоящее из векторов $J(k, h), k=\left(k_{1}, \ldots, k_{d}\right) \in \mathbb{Z}^{n}$, с компонентами $J_{j}(k, h)$, удовлетворяющими условию квантования Бора-Зоммерфельда-Маслова

$$
J_{j}(k, h)=\left(\frac{m_{j}}{4}+k_{j}\right) h, \quad J(k, h) \in \mathscr{M} .
$$

Тогда для таких $J(k, h)$ с точностью до $O(h)$ (в подходящей норме) определен канонический оператор Маслова [1], [2]

$$
K_{\Lambda(J(k, h))}^{h}: C^{\infty}\left(\Lambda\left(J^{k}(h)\right) \mapsto C^{\infty}(M),\right.
$$

и по каждой функции $\chi \in C^{\infty}\left(\Lambda(J)\right.$ можно построить функции $u_{k}(x, h)=K_{\Lambda(J(k, h))}^{h} \circ \chi$. Функции $u_{k}(x, h)$ локализованы в окрестности проекций $\pi_{x} \Lambda(J(k, h))$ на многообразие $M$ : при $h \rightarrow+0 \operatorname{supp} u_{k}(x, h) \rightarrow \pi_{x} \Lambda\left(J^{k}(h)\right)$. Границы $\pi_{x} \Lambda(J(k, h))$ состоят из поверхностей (кривых), называемых каустиками.

Семейства лагранжевых торов, их параметризация переменными действия, канонический оператор Маслова на них и т.д. определяются независимо от гамильтоновых систем и дифференциальных операторов. Рассмотрим $h$-псевдодифференциальный самосопряженный оператор

$$
\widehat{L}=L\left(x,-i h \frac{\partial}{\partial x} ; h\right),
$$

заданный своим вейлевским символом $L(x, p ; h)$, принадлежащим соответствующему классу функций на $T^{*} M$ (см. [1], [3]) и имеющим разложение

$$
L=H(x, p)+h L_{1}(x, p)+h^{2} L_{2}(x, p)+\cdots .
$$

Работа выполнена при поддержке Российского фонда фундаментальных исследований (грант № 08-01-00726) и фонда CNRS.

(C) С. Ю. Доврохотов, М. Руло, 2010 
с параметром $h$. Главный символ - функция $H(x, p)$ - называется гамильтонианом; для упрощения изложения считаем, что $L_{1}(x, p)=0$ (от этого предположения можно отказаться). Рассмотрим теперь семейство лагранжевых многообразий, инвариантных по отношению к векторному полю $X_{H}$, порожденному гамильтонианом $H$. Тогда описанная выше процедура позволяет построить "асимптотические" собственные функции $u^{k}(x, h)$ и значения $E^{k}(h)$ в том смысле, что $\left(\widehat{L}-E^{k}(h)\right) u^{k}(x, h)=\mathscr{O}()$. Последовательности $u^{k}(x, h), E^{k}(h)$ часто называют "квазимодами" или спектральными сериями.

Семейства лагранжевых торов, описанных выше, может быть построено, например, в случае, когда гамильтонова система, задаваемая $H$, вполне интегрируема, и $\Lambda(J)=$ $((x, p)=X(\varphi, J), P(\varphi, J))$ - связные компактные компоненты максимальной размерности в $T^{*} M$ отображения момента $m$, которое предполагается регулярным на некотором $\Lambda_{0}=\Lambda\left(I^{0}\right)$, лежащем на линии уровня $H=E$. Тогда $\Lambda(J)$ - лиувиллевы торы, инвариантные относительно фазового потока, порожденного гамильтонианом $H$. Выбирая функции $\chi$ на $\Lambda(J)$ равными 1 и полагая $E^{k}(h)=E(J(k, h)$, мы получаем множество квазимод ("спектральную серию") $u^{k}(x, h), E^{k}(h)$ оператора $\widehat{L}$, отвечающих семейству $\Lambda(J)[1]$. Предположение о гладкой зависимости семейства $\Lambda(J)$ от параметров $J$ (в обычном смысле) не обязательно, сказанное остается в силе при соответствующем изменении множества значений $\{J(k, h)\}$; в частности, вместо лиувиллевых торов можно рассматривать KAM-торы, возникающие в том случае, когда гамильтониан $H$ представляет собой возмущение некоторого интегрируемого гамильтониана. Таким ситуациям вместе с вопросами о полноте квазимод или "мощности" спектральной серии (по отношению к полному спектру оператора $\widehat{L}$ ) посвящены глубокие исследования, проведенные в [4], [5].

Требование интегрируемости гамильтоновой системы является весьма жестким. Цель этой заметки - показать, используя соображения близкие к [6], что некоторые спектральные серии можно получить в случае существования у гамильтоновой системы лишь одного (индивидуального) лагранжева тора с почтипериодическим или периодическим движением, и объяснить, как такие торы получить с помощью принципа Мопертюи-Якоби.

\section{2. Почти инвариантные торы и квазиклассические спектральные серии псевдодифференциальных операторов с параметром.}

ОПредЕлениЕ 1 . Пусть $N$ - некоторое натуральное число, $X_{H}$ - гамильтоново векторное поле на $T^{*} M$, заданное гладким гамильтонианом $H$, и в некоторой окрестности лагранжева тора $\Lambda_{0}$ задано параметризованное переменными действия $J$ гладкое семейство лагранжевых торов $\Lambda_{N}^{J}$ с квазипериодическим или периодическим движением на них. Назовем $\Lambda_{N}^{J}$ почти инвариантными $\bmod O\left(\left|J-I^{0}\right|^{N}\right)$ торами векторного поля $X_{H}$, если его траектории на $\Lambda^{J}$ удовлетворяют соответствующей гамильтоновой системе с точностью до $O\left(\left|J-I^{0}\right|^{N}\right)$ и

$$
\left.H\right|_{\Lambda^{J}}=H^{N}\left(J, I^{0}\right)+O\left(\left|J-I^{0}\right|^{N+1}\right),
$$

где $H^{N}\left(J, I^{0}\right)$ - некоторая гладкая функция.

Предположим, что фазовый поток, задаваемый гладким гамильтонианом $H$, на некотором лагранжевом торе $\Lambda=\Lambda_{0} \in T^{*} M$ имеет форму квазипериодического или периодического движения

$$
(P(t), X(t))=Y^{0}\left(\omega^{0} t+\varphi\right), \quad Y^{0}(\varphi)=(P(\varphi), X(\varphi))
$$

(выполнения какого-либо условия о несоизмеримости компонент вектора частот $\omega^{0}$ здесь не требуется). Обозначим через $I^{0} d$-мерный вектор, состоящий из действий на этом торе. Введем $2 d \times d$ матрицу $Y_{\varphi}^{0}=\left(\partial Y^{0} / \partial \varphi_{1}, \ldots, \partial Y^{0} / \partial \varphi_{d}\right)$ и $d \times d$ симметричную матрицу $M=-\left({ }^{t} Y_{\varphi}^{0} Y_{\varphi}^{0}\right)^{-1}$. Далее обозначим через $\mathbf{J}$ стандартную симплектическую матрицу

$$
\mathbf{J}=\left(\begin{array}{cc}
0 & -\mathbf{I d}_{d} \\
\mathbf{I} \mathbf{d}_{d} & 0
\end{array}\right)
$$


где $\mathbf{I} \mathbf{d}_{d}-d \times d$ единичная матрица, и положим $Y^{J}=\mathbf{J} Y_{\varphi}^{0} M$. Определим $d$-мерную вектор-функцию $q(J, \phi)$ как решение системы квадратных уравнений

$$
q_{j}-\frac{1}{2}\left\langle q,{ }^{t} Y_{I}^{0} \widehat{J} Y_{J \varphi_{j}}^{0} q\right\rangle=J_{j}-I_{j}^{0}, \quad j=1,2, \ldots, d,
$$

фиксированное условием $q=J-I+O\left(\left(J-I^{0}\right)^{2}\right)$ при $J \rightarrow I^{0}$.

Теорема 1 [7]. 1) Уравнения

$$
y=Y(J, \phi) \equiv{ }^{t}(P(J, \varphi), X(J, \varphi)) \equiv Y^{0}(\varphi)+Y_{I}^{0}(\varphi) q(\varphi, J)
$$

определяют почти инвариантные $\bmod O\left(\left|J-I^{0}\right|\right)$ лагранжевы торы $\Lambda_{1}$ векторного поля $X_{H}$, движение на которых задается вектором частот $\omega^{0} u$

$$
\left.H\right|_{\Lambda_{1}}=E+\left\langle\omega^{0}, J-I^{0}\right\rangle+O\left(\left(J-I^{0}\right)^{2}\right) .
$$

2) Пусть вектор частот $\omega^{0}=\left(\omega_{1}^{0}, \ldots, \omega_{d}^{0}\right)$ удовлетворяет диофантовому условию. Тогда для любого натурального $N$ в окрестности $\Lambda_{0} \subset\{H=E\}$ существует гладкое каноническое преобразование $\kappa_{N}:(J, \varphi) \mapsto\left(J^{\prime}, \varphi^{\prime}\right)$ и полином $H_{N}(\iota)$ степени $N$ аргумента $\iota=J^{\prime}-I^{0}$ maкuе, чmо

$$
H \circ \kappa \circ \kappa_{N}\left(J^{\prime}, \varphi^{\prime}\right)=H_{N}(\iota)+\mathscr{O}\left(\iota^{N+1}\right)
$$

и уравнения $y=Y \circ \kappa \circ \kappa_{N}\left(J^{\prime}, \varphi^{\prime}\right)$ определяют почти инвариантные $\bmod O\left((\iota)^{N}\right)$ лагранжевы торы $\Lambda_{N}^{J^{\prime}}$ поля $X_{H}$. При этом $J^{\prime}$ суть переменные действия, вычисленные на ииклах $\gamma_{j}$, которые также задают не зависящие от $J^{\prime}$ индексы Маслова $m_{j}, j=1, \ldots, d$.

ЗАмечАние 1. Это утверждение дает в точности нормальные формы Бирхгофа. Факт существования почти инвариантных лагранжевых торов вытекает из теоремы ДарбуВайнстейна, справедливой для общих лагранжевых многообразий [8]. Построения, данные в теореме 1, дают ее простое доказательство и достаточно явные формулы в рассматриваемом случае.

Теорема 2. Пусть $\Lambda^{0}$ - как и ранее (гладкое) лагранжево многообразие лежащее на энергетическом уровне $E:\left.H\right|_{\Lambda^{0}}=E$. Пусть заданы некоторые натуральное $N u$ вещественное $\delta, 0 \leqslant \delta \leqslant 1$, ири $N \geqslant 2$ выполнено диофантово условие для вектора частот $\omega$ (при $N=\delta=1$ выполнение диофантово условия не предполагается). Пусть далее $\Lambda_{N}^{J}$ - построенное в теореме 1 семейство почти инвариантных лагранжевых торов с ииклами $\gamma_{j}$, индексами $m_{j}$ и инвариантной мерой $d \varphi_{1}^{0} \ldots d \varphi_{d}^{0}$. Определим для каждого фиксированного $h$ множество векторов $J(k, h), k=\left(k_{1}, \ldots, k_{d}\right) \in \mathbb{Z}^{d}, c$ компонентами $J_{j}(k, h)=h\left(m_{j} / 4+k_{j}\right), j=1,2, \ldots, d$, где $k$ выбраны таким образом, что $\left|J(k, h)-I^{0}\right|=h^{\delta}$. Положим

$$
E(k, h)=E+H_{N}\left(J(k, h)-I^{0}\right) \equiv E+\left\langle\omega, J(k, h)-I^{0}\right\rangle+O\left(\left|J(k, h)-I^{0}\right|^{2}\right)
$$

$u u_{k}=K_{\Lambda_{N}^{J(k, h)}}^{h} \circ 1$.

Тогда $\left(u_{k}(x, h), E_{k}(h)\right)$ суть квазимоды оператора $\widehat{L}=H(x,-i h \partial / \partial x)+\mathscr{O}\left(h^{2}\right)$ :

$$
\left(\widehat{L}-E_{k}(h)\right) u_{k}(x, h)=\mathscr{O}\left(h^{1+\delta}\right)
$$

и расстояние от любого $E_{k}(h)$ до спектра оператора $\widehat{L}$ не превосходит $\mathscr{O}\left(h^{2}\right)$. В частности, если спектр оператора $\widehat{L}$ дискретен, то найдется собственное значение $\widetilde{E}$ оператора $\widehat{H}$ такое, что $\widetilde{E}=E_{k}(h)+\mathscr{O}\left(h^{2}\right)$. Построенные квазимоды имеют относительно максимальную плотность в $h^{\delta}$-окрестности $\Lambda^{0}$ (см. [4], [5]). 
3. Построение почти инвариантных торов с помощью принципа Мопертюи-Якоби. Нахождение даже одного инвариантного тора не интегрируемой гамильтоновой системы относится к числу открытых задач. Покажем, как при ее решении можно использовать известный принцип Мопертюи-Якоби [9]-[11].

Пусть $(\mathscr{H}(p, x), H(p, x))$ - пара функций Гамильтона на $T^{*} M$, которые допускают общую регулярную энергетическую поверхность $\Sigma$ со значениями энергии $(\mathscr{E}, E)$. Тогда известно, что траектории соответствующих гамильтоновых систем на энергетических уровнях $(\mathscr{E}, E)$ связаны равенствами $(\mathscr{P}(\tau), \mathscr{X}(\tau))=(P(t(\tau)), X(t(\tau)))$ с репараметризацией времени $t=t(\tau)$, определяемой уравнением $d t / d \tau=\mathscr{G}(\tau)$ с некоторой гладкой функцией $\mathscr{G}$, зависящей от начальных условий (см. [10; теорема 3.7.7]). B этом случае пара $(\mathscr{H}, H)$ удовлетворяет принципу Мопертюи-Якоби на уровнях энергии $(\mathscr{E}, E)$.

Для наших целей интересной является ситуация, когда вектор-функции $(\mathscr{P}(\tau), \mathscr{X}(\tau))$ задают квазипериодическое (или периодическое) движение с ( $d$-мерным) вектором частот $\widetilde{\omega}$ на $d$-мерном (лагранжевом) торе

$$
\Lambda_{0} \subset T^{*} M, \quad \Lambda_{0}=\left\{p=P^{0}(\varphi), x=X^{0}(\varphi)\right\},
$$

где $P^{0}(\varphi), X^{0}(\varphi)-2 \pi$-периодические функции по каждой фазе $\varphi_{j}$. В этом случае

$$
(\mathscr{P}(\tau), \mathscr{X}(\tau))=\left(P^{0}(\widetilde{\omega} \tau+\varphi), X^{0}(\widetilde{\omega} \tau+\varphi)\right)
$$

и $\Lambda_{0}$ - инвариантный лагранжев гамильтоновой системы с гамильтонианом $\mathscr{H}$. Этот тор является инвариантным и для гамильтоновой системы с гамильтонианом $H$, но с несколько другой динамикой.

Теорема 3. Пусть $(\mathscr{H}, H)$ удовлетворяет приниипу Мопертюи-Якоби с энергиями $(\mathscr{E}, E)$ и $\Lambda_{0}-$ инвариантный тор гамильтоновой системы с гамильтонианом $\mathscr{H}$. Предположим, что частоты $\widetilde{\omega}$ удовлетворяют диофантовым условиям, и пусть $(\tau, t)$ связаны равенством $d t=G\left(\widetilde{\omega} \tau+\varphi_{j}^{0}\right) d \tau$ с гладкой функиией $G\left(\varphi^{0}\right)=\left.\mathscr{G}\right|_{\Lambda^{0}}, 2 \pi$-периодической по каждой фазе $\varphi_{j}^{0}$. Тогда можно определить репараметризачию $\Lambda_{0}$ равенством

$$
\Phi: \varphi_{0} \mapsto \varphi=\Phi\left(\varphi_{0}\right)=\varphi_{0}+\widetilde{\omega} f\left(\varphi_{0}\right),
$$

где $f$ - гладкая периодическая функиия с нулевым средним $\langle f\rangle=0$, при этом движение на $\Lambda^{0}$, порожденное гамильтонианом $H$, при такой параметризачии будет квазипериодическим с вектором частот $\omega=\widetilde{\omega} /\langle G\rangle$ u $\operatorname{det}\left(\partial \Phi / \partial \varphi_{0}\left(\varphi_{0}\right)\right)=\langle G\rangle / G$. Здесъ $\langle G\rangle$ естъ среднее от $G$ по тору $\Lambda_{0}$.

Ситуация, описанная в теореме 3 , имеет место, например, в случае, когда гамильтонова система, задаваемая гамильтонианом $\mathscr{H}$, вполне интегрируема и $\Lambda_{0}-$ некоторый лиувиллев тор векторного поля $X \mathscr{H}$ с почтипериодическим движением с вектором частот $\widetilde{\omega}$. Заметим, что нет никакой причины, в силу которой гамильтонова система с гамильтонианом $H$ в общем случае была бы также вполне интегрируемой или близкой к вполне интегрируемой. Но если предположить, что вектор частот $\widetilde{\omega}$ удовлетворяет диофантовым условиям, то применяя последовательно теоремы 3,1 и 2 , мы получаем инвариантный тор векторного поля $X_{H}$ с почтипериодическим движением, затем семейство почти инвариантных торов и спектральную серию соответствующего (псевдо) дифференциального оператора, порожденную этим семейством. Если в теореме 2 ограничиться случаем $N=1$, то угловые переменные $\varphi$ на почти инвариантных торах, сопряженные с $J$, совпадут с угловыми переменными на торе $\Lambda^{0}$. Тогда

$$
d \varphi_{1} \ldots d \varphi_{d}=\frac{\langle G\rangle}{G\left(\varphi^{0}\right)} d \varphi_{1}^{0} \ldots d \varphi_{d}^{0}
$$

Поэтому в каноническом операторе Маслова, определяющем собственные функции $\widehat{L}$, можно использовать угловые переменные $\varphi_{j}^{0}$ (связанные с гамильтонианом $\mathscr{H}$ ), заменив функцию 1 на $\sqrt{G\left(\varphi_{0}\right)}$. 
Напомним один из основных примеров, в котором применяется принцип соответствия Мопертюи-Якоби. Предположим, что на многообразии $M$ с гладкой Римановой метрикой $d s^{2}=\sum_{1 \leqslant i, j \leqslant d} g_{i j}(x) d x^{i} \otimes d x^{j}$ задан гладкий потенциал $V$, причем $E>\sup _{M} V$. Тогда гамильтонианы

$$
\mathscr{H}=\frac{1}{2(E-V)} \sum_{1 \leqslant i, j \leqslant d} g^{i j}(x) p_{i} p_{j}, \quad H=\frac{1}{2} \sum_{1 \leqslant i, j \leqslant d} g^{i j}(x) p_{i} p_{j}+V(x)
$$

удовлетворяют принципу Мопертюи-Якоби на уровнях энергии $(1, E)$, при этом времена $\tau$ и $t$ связаны равенством $d \tau=(E-V) d t$ и $G=1 /\left(E-V\left(X^{0}\left(\varphi^{0}\right)\right)\right)$.

4. Приложение к теории волн на воде. Рассмотрим случай, когда $\mathscr{H}(x, p)=$ $g(x) p^{2}$ задается метрикой $g(x)$ Лиувилля в некоторой области $\Omega$ на двумерной плоскости, диффеоморфной кольцу. Все интегрируемые ситуации в случае, когда второй интеграл в инволюции квадратичен по импульсам $p_{1}, p_{2}$, полностью изучены. Комментарии и библиографию можно найти в [12], а также в работе [13], в которой соответствующие лиувиллевы торы были использованы для построения квазимод оператора $\nabla g(x) \nabla$, описывающих волны на воде, захваченные донными неоднородностями в длинноволновом приближении. Используя эти результаты и квазиклассический аналог принципа Мопертюи-Якоби, сконструируем профили дна бассейна, которые порождают серии квазимод полного псевдодифференциального оператора волн на воде $\widehat{L}=L(-i \partial / \partial x, x ; h)$ с вейлевским символом

$$
L(p, x ; h)=H(p, x)+h^{2} L_{2}+\cdots .
$$

Здесь $H(p, x)=|p| \tanh (D(x)|p|)$ и заданная в области $\Omega$ гладкая функция $D(x)$ описывает дно бассейна (см. [2]).

Зафиксируем некоторое положительное $E$, умножим равенство на $H(p, x)=E$ на $D(x)$ и положим $z=|p| D(x), \phi\left(z^{2}\right)=z \tanh z$. Обозначим через $\psi: \mathbb{R}_{+} \rightarrow \mathbb{R}_{+}$отображение, обратное к $\phi\left(z^{2}\right)$; оно определяется гладкой строго возрастающей функцией, поэтому равенство $H(p, x)=E$ для фиксированного $E$ может быть переписано в виде

$$
\mathscr{H}(x, p)=g(x, E) p^{2}=1, \quad g(x, E)=\frac{D^{2}(x)}{\psi(E D(x))} .
$$

На поверхности энергии $\mathscr{H}(x, p)=1$ гамильтоновы векторные поля $X_{H}$ и $X_{\mathscr{H}}$ связаны равенством

$$
X_{\mathscr{H}}=G^{\prime} X_{H}, \quad G^{\prime}=\frac{\psi(E D(x))}{D(x) \psi^{\prime}(E D(x))},
$$

которое и индуцирует репараметризацию траекторий $d t=G d \tau$, соответствующую принципу Мопертюи-Якоби. Введем отображение $\chi: \mathbb{R}_{+} \rightarrow[0,1[$, заданное гладкой возрастающей функцией $\chi(D)=D^{2} / \psi(D)$, и выберем функцию $D(x)=D(x, E)$, задающую дно бассейна, как решение уравнения

$$
g(x, E)=g(x)=E^{-2} \chi(E D(x, E)),
$$

где $g(x)$ - метрика Лиувилля.

Теорема 4. Пусть метрика $g(x, E)$ выбрана (как и в [13]) так, что геодезический поток $\mathscr{H}(x, p)=g(x, E) p^{2}$ интегрируем и допускает лиувиллевы торы с векторами частот $\widetilde{\omega}$, удовлетворяющими диофантовому условию. Зафиксируем один из этих торов $\Lambda_{0}$. Тогда для любого $E>0$ и этой лиувиллевой метрики уравнение

$$
g(x)=E^{-2} \chi(E D(x, E))
$$

определяет "функиию дна" $D(x)=D(x, E)$ на $\Omega$ такую, что $\Lambda_{0}$ есть инвариантный лагранжев тор пары гамильтонианов $(H, \mathscr{H})$ с квазипериодическими траекториями 
с энергиями $(E, 1)$. Этот тор $\Lambda_{0}$ порождает последовательность квазимод оператоpa $\widehat{L}$ с энергиями (квадратами частот захваченных мод)

$$
E+\frac{\left\langle\omega, J(k, h)-I^{0}\right\rangle}{\left\langle G^{\prime}\right\rangle}+O\left(\left|J(k, h)-I^{0}\right|^{2}\right),
$$

где $J(k, h), I^{0}$ определены в теоремах 1,2 и $\left\langle G^{\prime}\right\rangle$ есть среднее от $G^{\prime}$ на торе $\Lambda_{0}$.

\section{СПИСОК ЦИТИРОВАННОЙ ЛИТЕРАТУРЫ}

[1] В.П. Маслов, М.В. Федорюк, Квазиклассическое приближение для уравнений квантовой механики, Наука, M., 1976. [2] S. Yu. Dobrokhotov, P. N. Zhevandrov, Russ. J. Math. Phys., 10:1 (2003), 1-31. [3] V. Ivrii, Microlocal Analysis and Precise Spectral Asymptotics, Springer Monogr. Math., Springer-Verlag, Berlin, 1998. [4] V.F. Lazutkin, KAM Theory and Semi-Classical Approximation to Eigenfunctions, Ergeb. Math. Grenzgeb. (3), 24, Springer-Verlag, Berlin, 1993. [5] Y. Colin de Verdiére, Invent. Math., 43 (1977), 15-52. [6] А. Д. Крахнов, УМН, 31:3 (1976), 217-218. [7] В. В. Белов, С. Ю. Доброхотов, B. А. Максимов, ТМФ, 135:3 (2003), 378-408. [8] S. Bates, A. Weinstein, Lectures on the Geometry of Quantization, Berkeley Math. Lect. Notes, 8, Amer. Math. Soc., Providence, RI, 1997. [9] В. И. Арнольд, Математические методы классической механики, Наука, М., 1974. [10] R. Abraham, J.E. Marsden, Foundations of Mechanics, Benjamin/Cummings Publ., Reading, MA, 1978. [11] A. V. Tsiganov, J. Nonlinear Math. Phys., 8:1 (2001), 157-182. [12] B. A. Dubrovin, A. T. Fomenko, S. P. Novikov, Modern Geometry - Methods and Applications. Vol. 1. The Geometry of Surfaces, Transformation Groups, and Fields, Grad. Texts in Math., 93, Springer-Verlag, New York, NY, 1984. [13] В. С. Матвеев, Матем. заметки, 64:3 (1998), 414-422.

\section{С. Ю. Доброхотов}

Институт проблем механики РАН, Московский физико-технический институт (государственный университет) E-mail: dobr@ipmnet.ru

\section{М. Руло}

Centre de Physique Théorique, Université du Sud Toulon-Var

E-mail: rouleux@cpt.univ-mrs.fr
Поступило

01.09.2009

Исправленный вариант

27.10.2009 